\title{
Normal stress differences and beyond-Navier-Stokes hydrodynamics
}

\author{
Meheboob Alam ${ }^{1, \star}$ and Saikat Saha ${ }^{1}$ \\ ${ }^{1}$ Jawaharlal Nehru Centre for Advanced Scientific Research, Jakkur PO, Bangalore 560064, INDIA
}

\begin{abstract}
A recently proposed beyond-Navier-Stokes order hydrodynamic theory for dry granular fluids is revisited by focussing on the behaviour of the stress tensor and the scaling of related transport coefficients in the dense limit. For the homogeneous shear flow, it is shown that the eigen-directions of the second-moment tensor and those of the shear tensor become co-axial, thus making the first normal stress difference $\left(\mathcal{N}_{1}\right)$ to zero in the same limit. In contrast, the origin of the second normal stress difference $\left(\mathcal{N}_{2}\right)$ is tied to the 'excess' temperature along the mean-vorticity direction and the imposed shear field, respectively, in the dilute and dense flows. The scaling relations for transport coefficients are suggested based on the present theory.
\end{abstract}

\section{Introduction}

As in colloidal suspensions and polymeric fluids, the dry granular fluid possesses prominent non-Newtonian properties, like the normal stress differences, shear-thickening and shear-thinning; for example, the normal stress differences can be of the order of its isotropic pressure in a sheared dilute granular gas in contrast to its infinitesimal magnitude in its molecular counterpart $[1,2]$, see Fig. 1; both the first $\left(\mathcal{N}_{1}\right)$ and second $\left(\mathcal{N}_{2}\right)$ normal stress differences can change sign depending on the mean density of the granular fluid [3-5]. Such order-one effects must be incorporated in the theoretical modelling of granular fluids so that the theory remains valid from the dilute to the dense limit. Recently we outlined a Grad-like [6] extended hydrodynamic theory for granular fluids [7-9], and derived analytical expressions for normal stress differences and other transport coefficients that are valid for (a) a large range of density (encompassing both the dilute and dense limits) and (b) small restitution coefficient (far away from the limit of elastic collisions). After a brief overview of this theory in Sec. 2, we analyse the stress tensor in the uniform shear flow of granular materials with a focus on the dense-limit behaviour of normal stress differences and their scaling relations as detailed in Secs. 3 and 4.

\section{Overview of 10-Moment Theory}

Going beyond the Navier-Stokes-order hydrodynamics of five field variables, here we consider an 'extended' set of 10 hydrodynamic fields: (i) the mass density $\rho(\boldsymbol{x}, t) \equiv$ $m n(\boldsymbol{x}, t)=m \int f(\boldsymbol{c}, \boldsymbol{x}, t) \mathrm{d} \boldsymbol{c}$, (ii) the coarse-grained velocity $\boldsymbol{u}(\boldsymbol{x}, t) \equiv\langle\boldsymbol{c}\rangle=\frac{1}{n(\boldsymbol{x}, t)} \int \boldsymbol{c} f(\boldsymbol{c}, \boldsymbol{x}, t) \mathrm{d} \boldsymbol{c}$, and (iii) the second moment tensor $\boldsymbol{M}(\boldsymbol{x}, t) \equiv\langle\boldsymbol{C C}\rangle=\frac{1}{n(\boldsymbol{x}, t)} \int \boldsymbol{C C} f(\boldsymbol{c}, \boldsymbol{x}, t) d \boldsymbol{c}$, where $n(\boldsymbol{x}, t)$ is the number density, $\boldsymbol{C} \equiv \boldsymbol{c}-\boldsymbol{u}$ is the

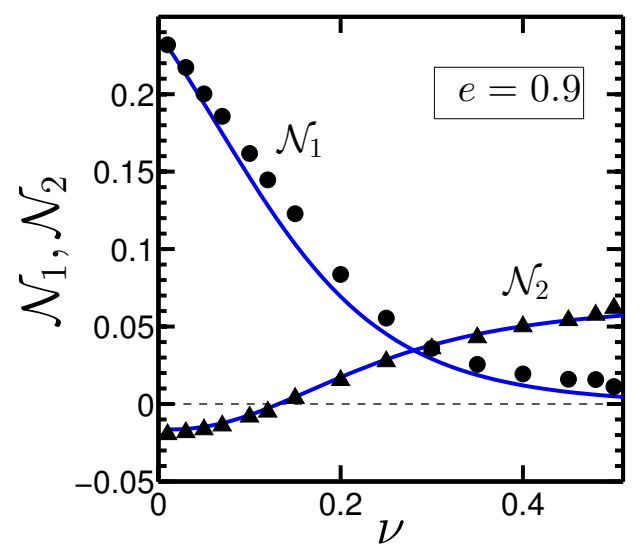

Figure 1. Variations of first $\left(\mathcal{N}_{1}\right)$ and second $\left(\mathcal{N}_{2}\right)$ normal stress differences with density for a restitution coefficient of $e=0.9$; the symbols refer to data from event-driven simulation and the lines refer to present theory. Adapted from Fig. 1 of Ref. [8].

peculiar velocity of particles and $f(\boldsymbol{c}, \boldsymbol{x}, t)$ is the singleparticle distribution function. The last hydrodynamic field $\boldsymbol{M}(\boldsymbol{x}, t)$ is required to account for normal stress differences $[7,8]$. The balance equations for the mass, momentum and second-moment, respectively, can be obtained by taking appropriate moment of the Enskog-Boltzmann equation:

$$
\begin{aligned}
& \frac{D \rho}{D t}=-\rho \boldsymbol{\nabla} \cdot \boldsymbol{u}, \\
& \rho \frac{D \boldsymbol{u}}{D t}=-\boldsymbol{\nabla} \cdot \boldsymbol{P}, \\
& \rho \frac{D \boldsymbol{M}}{D t}=-\boldsymbol{\nabla} \cdot \boldsymbol{Q}-\boldsymbol{P} \cdot \boldsymbol{\nabla} \boldsymbol{u}-(\boldsymbol{P} \cdot \boldsymbol{\nabla} \boldsymbol{u})^{T}+\boldsymbol{\aleph},
\end{aligned}
$$

where $D / D t=(\partial / \partial t+\boldsymbol{u} \cdot \boldsymbol{\nabla})$ is the material derivative, $\boldsymbol{P}$ is the stress tensor, given by

$$
\boldsymbol{P} \equiv \rho \boldsymbol{M}+\boldsymbol{\Theta}(m \boldsymbol{C}),
$$


$\boldsymbol{Q}$ is the flux of the second moment, a third-rank tensor, given by

$$
\boldsymbol{Q} \equiv \rho\langle\boldsymbol{C C C}\rangle+\boldsymbol{\Theta}(m \boldsymbol{C} \boldsymbol{C})
$$

and $\boldsymbol{N}$ is the collisional source of second moment, a second-rank tensor, given by

$$
\boldsymbol{\aleph} \equiv \boldsymbol{N}(m \boldsymbol{C} \boldsymbol{C})
$$

In Eqns. (4-5), the first and second terms refer to the kinetic and collisional contributions, respectively.

It is straightforward to show that the balance equation for the granular energy follows from the trace of (3),

$$
\frac{3}{2} \rho \frac{D T}{D t}=-\boldsymbol{\nabla} \cdot \mathbf{q}-\boldsymbol{P}: \boldsymbol{\nabla} \boldsymbol{u}-\mathcal{D},
$$

where $T \equiv M_{\alpha \alpha} / 3$ is the granular temperature,

$$
q_{\alpha} \equiv \frac{1}{2} Q_{\alpha \beta \beta}=\frac{1}{2} \rho M_{\alpha \beta \beta}+\frac{1}{2} \Theta_{\alpha \beta \beta}
$$

is the heat flux vector, and

$$
\mathcal{D} \equiv-\frac{1}{2} \boldsymbol{\aleph}_{\beta \beta}=-\frac{1}{2} \boldsymbol{N}\left(m C^{2}\right)
$$

is the rate of dissipation of kinetic energy per unit volume. The balance equations (1-2, 7), along with closure relations for (4), (8) and (9), constitute the Navier-Stokesorder hydrodynamics for which the equation for the deviatoric part of the second moment is satisfied identically.

For an extended hydrodynamic theory in terms of ten moments, the balance equation (3) for full second moment tensor along with mass and momentum balances (1-2) are needed. For a closure of (3), the deviatoric part of the third-order $Q_{\gamma \alpha \beta}$,

$$
\widehat{Q}_{\gamma \alpha \beta}=Q_{\gamma \alpha \beta}-\frac{1}{5}\left(Q_{\gamma \xi \xi} \delta_{\alpha \beta}+Q_{\alpha \xi \xi} \delta_{\gamma \beta}+Q_{\beta \xi \xi} \delta_{\alpha \gamma}\right),
$$

is assumed to be zero, leaving only its isotropic part, the heat flux vector (8), to be evaluated as a constitutive relation. Recently we showed [7] that the heat-flux follows a generalized Fourier law of the form

$$
\mathbf{q}=-\kappa \cdot \nabla T-\kappa_{\rho} \cdot \nabla \rho-\Psi: \nabla \widehat{M},
$$

where $\boldsymbol{\kappa}$ is the thermal conductivity tensor (of second rank) and $\boldsymbol{\kappa}_{\rho}$ is the Dufour tensor; the fourth-rank tensor $\boldsymbol{\Psi}$ is tied to non-equilibrium heat-flux due to the stress-gradient (note that $\widehat{\boldsymbol{M}}$ is the deviatoric part of the second moment which is proportional to kinetic shear stress and normal stress differences). Collectively, the anisotropic thermal conductivity $\boldsymbol{\kappa}$ and the stress-gradient conductivity $\boldsymbol{\Psi}$ are responsible for the "parallel" heat-flux (along the flow direction in the absence of temperature gradient); the latter is a well-known phenomenon in rarefied gases [6]. Closedform expressions of $\boldsymbol{\kappa}, \boldsymbol{\kappa}_{\rho}$ and $\boldsymbol{\Psi}$ for a dilute granular gas undergoing shear flow can be found in Ref. [7, 9].

The balance equations (1-3), along with closure relations for the stress tensor (4), the source of second moment (6) and the heat-flux vector (11), constitute the 10-moment theory for a granular fluid - this represents the minimal

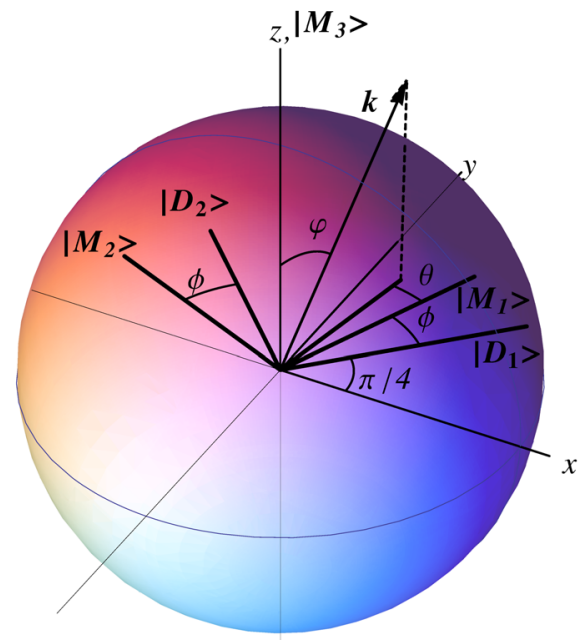

Figure 2. Schematic of the coordinate system displaying the eigen-basis of the shear tensor $\boldsymbol{D}$ and the second-moment tensor $\boldsymbol{M}$. Note that the contact vector $\boldsymbol{k}$ makes an angle $\varphi$ with $\left|M_{3}\right\rangle$, and $\theta$ is the angle between $\left|M_{1}\right\rangle$ and $\boldsymbol{k}-(\boldsymbol{k} \cdot \boldsymbol{z}) \boldsymbol{z}$, the projection of $\boldsymbol{k}$ on the shear plane. The uniform shear flow, $\boldsymbol{u}=(2 \dot{\gamma} y, 0,0)$, is directed along the $x$-direction, with the velocity gradient along the $y$-direction and the mean-vorticity along the $z$-direction. Adapted from Fig. 2 of Ref. [8].

continuum model that incorporates (i) normal stress differences as well as (ii) anisotropic heat flux [7, 8]. In the remainder of this paper, we will analyse the dense-limit behaviour of the stress tensor and related transport coefficients for uniform shear flow for which the anisotropic Gaussian [2, 7, 8] serves as the reference distribution function. The latter follows from the maximum entropy principle.

\section{Uniform Shear Flow (USF) and the Second-moment Balance}

The coordinate system is depicted in Fig. 2, with the uniform shear flow (USF) occurring in the $(x, y)$-plane; the $x$ and $y$ denote flow and gradient directions, respectively, and the $z$ direction is perpendicular to $x-y$ plane. The velocity gradient tensor,

$$
\boldsymbol{\nabla} \boldsymbol{u}=\left[\begin{array}{ccc}
0 & 2 \dot{\gamma} & 0 \\
0 & 0 & 0 \\
0 & 0 & 0
\end{array}\right] \equiv \boldsymbol{D}+\boldsymbol{W}
$$

completely characterizes the uniform shear flow $(2 \dot{\gamma}$ is the overall shear rate), with $\boldsymbol{D}$ and $\boldsymbol{W}$ denoting the shear and spin tensors, respectively. It is straightforward to verify that $\dot{\gamma},-\dot{\gamma}$ and 0 are the eigenvalues of $\boldsymbol{D}$ and the corresponding orthonormal eigenvectors are, respectively,

$$
\left|D_{1}\right\rangle=\left[\begin{array}{c}
\cos \frac{\pi}{4} \\
\sin \frac{\pi}{4} \\
0
\end{array}\right],\left|D_{2}\right\rangle=\left[\begin{array}{c}
-\sin \frac{\pi}{4} \\
\cos \frac{\pi}{4} \\
0
\end{array}\right],\left|D_{3}\right\rangle=\left[\begin{array}{l}
0 \\
0 \\
1
\end{array}\right] .
$$


While $\left|D_{3}\right\rangle$ is directed along the $z$-axis, the shear-plane eigenvectors $\left|D_{1}\right\rangle$ and $\left|D_{2}\right\rangle$ are rotated by $45^{\circ}$ anticlockwise from the $x y$-axes.

\subsection{Construction of second moment tensor}

We can decompose the second moment tensor $\boldsymbol{M}$ into an isotropic tensor and a traceless deviatoric part $\boldsymbol{M}=$ $T\left[\delta_{\alpha \beta}\right]+\widehat{\boldsymbol{M}}$, where $\widehat{\boldsymbol{M}} / T$ is the dimensionless counterpart of its deviatoric tensor whose eigenvalues $\xi, \varsigma$ and $\zeta$ satisfy $\xi+\varsigma+\zeta=0$. Therefore the eigenvalues of $\boldsymbol{M}$ are $T(1+\xi), T(1+\varsigma)$ and $T(1+\zeta)$, with the corresponding orthonormal set of eigen-directions being denoted by $\left|M_{1}\right\rangle$, $\left|M_{2}\right\rangle$ and $\left|M_{3}\right\rangle$, respectively. The second-moment tensor can be expressed as

$\boldsymbol{M}=T(1+\xi)\left|M_{1}\right\rangle\left\langle M_{1}|+T(1+\varsigma)| M_{2}\right\rangle\left\langle M_{2}|+T(1+\zeta)| M_{3}\right\rangle\left\langle M_{3}\right|$.

Referring to Fig. 2, we assume that the shear-plane eigenvectors $\left|M_{1}\right\rangle$ and $\left|M_{2}\right\rangle$ can be obtained by rotating the system of axes at an angle $(\pi / 4+\phi) \equiv \bar{\phi}$, with the angle $\phi$ being unknown, in the anti-clockwise sense about the $z$-axis which coincides with $\left|M_{3}\right\rangle$ :

$$
\left|M_{1}\right\rangle=\left[\begin{array}{c}
\cos \bar{\phi} \\
\sin \bar{\phi} \\
0
\end{array}\right],\left|M_{2}\right\rangle=\left[\begin{array}{c}
-\sin \bar{\phi} \\
\cos \bar{\phi} \\
0
\end{array}\right],\left|M_{3}\right\rangle=\left[\begin{array}{l}
0 \\
0 \\
1
\end{array}\right]
$$

Inserting (15) into (14), we obtain the following expression for deviator of the second moment tensor

$$
\widehat{\boldsymbol{M}}=T\left[\begin{array}{ccc}
\lambda^{2}+\eta \sin 2 \phi & -\eta \cos 2 \phi & 0 \\
-\eta \cos 2 \phi & \lambda^{2}-\eta \sin 2 \phi & 0 \\
0 & 0 & -2 \lambda^{2}
\end{array}\right]
$$

where we have introduced the following notations

$$
\eta \equiv \frac{1}{2}(\varsigma-\xi) \geq 0 \quad \text { and } \quad \lambda^{2} \equiv \frac{1}{2}(\varsigma+\xi)=-\frac{\zeta}{2} \geq 0 .
$$

Note that while $\eta \sim\left(T_{x}-T_{y}\right)$ is a measure of the anisotropy of the second-moment tensor in the shear plane, $\lambda^{2}$ is a measure of the excess temperature [8] $\left[T^{e x}=\left(T-T_{z}\right)\right.$, where $T_{i}$ is the temperature along the $i$-th direction] along the mean vorticity direction. It is straightforward to verify that the eigenvalues in the shear-plane can be expressed in terms of $\eta$ and $\lambda$ via

$$
\xi=\lambda^{2}-\eta \quad \text { and } \quad \varsigma=\lambda^{2}+\eta>\xi,
$$

with the eigenvalue, $\zeta$, along the vorticity direction $(z)$, being given by Eq. (17).

\subsection{The balance of second moment in USF}

In the steady uniform shear flow, the number density $n$, the velocity gradient $\boldsymbol{\nabla} \boldsymbol{u}$ and the components of $\boldsymbol{M}$ are constants and the contracted third-moment vanishes. Therefore, the mass and momentum balance equations, (1) and (2), are identically satisfied. The remaining balance equation (3), $P_{\delta \beta} u_{\alpha, \delta}+P_{\delta \alpha} u_{\beta, \delta}=\boldsymbol{\aleph}_{\alpha \beta}$, for the second-moment simplifies to

$$
\begin{aligned}
\rho M_{\delta \beta}\left(D_{\alpha \delta}+W_{\alpha \delta}\right) & +\rho M_{\delta \alpha}\left(D_{\beta \delta}+W_{\beta \delta}\right)+\Theta_{\delta \beta} D_{\alpha \delta} \\
& +\Theta_{\delta \alpha} D_{\beta \delta}=A_{\alpha \beta}+\widehat{E}_{\alpha \beta}+\widehat{G}_{\alpha \beta},
\end{aligned}
$$

where we made use of $u_{\alpha, \delta}=\left(D_{\alpha \delta}+W_{\alpha \delta}\right), P_{\alpha \beta}=\rho M_{\alpha \beta}+$ $\Theta_{\alpha \beta}$ and the following decomposition for the collisional source of second moment:

$$
\boldsymbol{\aleph}_{\alpha \beta}=A_{\alpha \beta}+\widehat{E}_{\alpha \beta}+\widehat{G}_{\alpha \beta}+\Theta_{\alpha \delta} W_{\beta \delta}+\Theta_{\beta \delta} W_{\alpha \delta} .
$$

The Savage-Jeffrey number [10], or, the dimensionless shear-rate is defined as

$$
R \equiv \frac{\dot{\gamma}}{4 \sqrt{T / \sigma^{2}}}=\left(\frac{\sqrt{T}}{\sigma \dot{\gamma} / 4}\right)^{-1} \equiv \frac{v_{s h}}{v_{t h}} .
$$

This parameter, which can also be interpreted as the inverse of the square root of dimensionless temperature, is a measure of the mean shear velocity $\left(v_{s h}=\sigma \dot{\gamma} / 2\right.$ over a particle diameter) relative to the thermal velocity $\left(v_{t h} \propto\right.$ $\sqrt{T}$ ) associated with the random motion of particles. The second-moment tensor $(14,16)$ in USF, constructed from its eigen-basis, is completely determined when $R, \eta, \phi$ and $\lambda^{2}$ are specified. Therefore the second-moment balance (19) can now be solved in terms of above four parameters for specified values of (i) the mean density $v$ and (ii) the restitution coefficient $e$.

\section{Dense Limit: Solution and Discussion}

The perturbation solution of equation (19), along with the resulting analytical expressions for the normal stress differences and other transport coefficients (shear viscosity, pressure, cooling rate, etc), have been detailed in Ref. [8]. Figure 1 illustrates the accuracy of theoretical predictions for two normal stress differences,

$$
\mathcal{N}_{1}=\left(P_{x x}-P_{y y}\right) / p, \quad \text { and } \quad \mathcal{N}_{2}=\left(P_{y y}-P_{z z}\right) / p,
$$

where $p=P_{\alpha \alpha} / 3$ is the mean pressure, via a comparison with particle simulation data.

Here we determine an approximate solution of the second-moment balance equation in the dense limit $(v \rightarrow$ $\left.v_{\max }\right)$. Since the collisional mechanism of momentum transfer dominates over its kinetic contribution as $v \rightarrow$ $v_{\max }$, the stress tensor can be approximated by its collisional contribution, i.e. $P_{\alpha \beta}=\Theta_{\alpha \beta}$ which has an integral expression [8]. The balance equations (19) simplify to

$$
\begin{array}{r}
A_{x^{\prime} x^{\prime}}+A_{y^{\prime} y^{\prime}}+A_{z^{\prime} z^{\prime}}=-3 \widehat{\Gamma}_{z^{\prime} z^{\prime}} \\
2 \dot{\gamma}\left[\left(\Theta_{x^{\prime} x^{\prime}}-\Theta_{y^{\prime} y^{\prime}}\right) \cos 2 \phi-2 \Theta_{x^{\prime} y^{\prime}} \sin 2 \phi\right]=-3 \widehat{\Gamma}_{z^{\prime} z^{\prime}} \\
2 \dot{\gamma}\left(\Theta_{x^{\prime} x^{\prime}}+\Theta_{y^{\prime} y^{\prime}}\right) \cos 2 \phi=\left(\Gamma_{x^{\prime} x^{\prime}}-\Gamma_{y^{\prime} y^{\prime}}\right) \\
-\dot{\gamma}\left(\Theta_{x^{\prime} x^{\prime}}+\Theta_{y^{\prime} y^{\prime}}\right) \sin 2 \phi=\Gamma_{x^{\prime} y^{\prime}}
\end{array}
$$

where $\Gamma_{\alpha \beta}=A_{\alpha \beta}+\widehat{E}_{\alpha \beta}+\widehat{G}_{\alpha \beta}$, with each tensorial quantity having an analytic integral expression which is evaluated as infinite series [7,8] as functions of $\eta, \lambda, \phi$ and $R^{2}$.

\subsection{Perturbation solution in the dense limit}

Substituting the expressions [8] for the integrals $\left(\Theta_{x^{\prime} x^{\prime}}+\right.$ $\left.\Theta_{y^{\prime} y^{\prime}}\right)$ and $\Gamma_{x^{\prime} y^{\prime}}$ into the last equation of (23) yields $\phi=0$; the remaining three equations can be solved perturbatively. The leading-order solution of (23) is given by

$$
\eta=0=\phi, \lambda \quad 2=\frac{10(1-e)}{21(3-e)}, \quad R^{2}=\frac{15(1-e)}{16(1+3 e)} .
$$


Since $\eta, \phi \rightarrow 0$, the first normal stress difference $\left(\mathcal{N}_{1} \propto\right.$ $\eta \sin 2 \phi$ ) vanishes as $v \rightarrow v_{\max }$, but the second normal stress difference remains finite $\left(\mathcal{N}_{2} \propto \lambda^{2} \neq 0\right)$ in the dense limit. The solution (24) indicates that $R, \lambda \sim \sqrt{(} 1-e)$ and therefore both the shear-rate and the excess temperature scale with inelasticity as $\sqrt{(1-e)}$ in the dense limit.

The next-order corrections for $\eta, R^{2}, \lambda^{2}$ and $\phi$ have also been calculated and all transport coefficients ( $p, \mu$, $\mathcal{N}_{1}$ and $\mathcal{N}_{2}$ ) can be expressed as functions of $v$ and $e$ (in dimensionless form):

$$
\begin{gathered}
p^{*} \approx \frac{(1+e) v^{2} g_{0}}{110880 R^{2}}\left(3465+7392 R^{2}+\eta R \chi_{1} \cos 2 \phi\right) \\
\mu^{*} \approx \frac{(1+e) v^{2} g_{0}}{18480 \sqrt{\pi} R}\left[3 \left(616+77 \sqrt{\pi} \frac{\eta \cos 2 \phi}{R}\right.\right. \\
\left.+4 \lambda^{2}\left(44+8 \eta^{2}-11 \lambda^{2}+4 \lambda^{4}\right)\right)+128\left(11-4 \lambda^{2}\right) R^{2} \\
\left.-4 \eta^{2}\left(22+\left(11-12 \lambda^{2}\right)\left(1+2 \cos ^{2} 2 \phi\right)\right)\right], \\
\mathcal{N}_{1} \approx \frac{12\left(231-\frac{8}{\sqrt{\pi}} \eta R\left(11-12 \lambda^{2}\right) \cos 2 \phi\right) \eta \sin 2 \phi}{\left(231\left(15+32 R^{2}\right)+\eta R \chi_{1} \cos 2 \phi\right)}, \\
\mathcal{N}_{2} \approx \frac{6\left(33\left(32 R^{2}-7 \eta \sin 2 \phi+21 \lambda^{2}\right)+\eta R \chi_{2} \cos 2 \phi\right)}{\left(231\left(15+32 R^{2}\right)+\eta R \chi_{1} \cos 2 \phi\right)},
\end{gathered}
$$

where

$$
\begin{gathered}
\chi_{1}=\frac{8}{\sqrt{\pi}}\left(462+33 \eta^{2}-352 R^{2}-132 \lambda^{2}+99 \lambda^{4}\right) \\
\chi_{2}=\frac{8}{\sqrt{\pi}}\left(66+6 \eta^{2}-64 R^{2}-33 \lambda^{2}+18 \lambda^{4}+\eta\left(11-12 \lambda^{2}\right) \sin 2 \phi\right)
\end{gathered}
$$

and $g_{0}$ is the contact value of the pair-correlation function [11]. A graphical comparison (not shown) of the above two dense-limit solutions for the first and second normal stress differences with the respective exact numerical-solution of second moment equation confirms the accuracy of present analytical solutions. The mechanistic origin of $\mathcal{N}_{1}$ and $\mathcal{N}_{2}$ is evident from (27-28).

\subsection{Discussion}

Although the Enskog-Boltzmann equation is unlikely to hold close to the maximum packing $\left(v \rightarrow v_{\max }\right)$ limit, it is interesting to know the predictions of the present theory on two specific issues in the dense limit: (i) the co-linearity between the eigen-directions of the stress and shear tensors and (ii) the scaling of the transport coefficients.

\subsubsection{Are the stress and shear tensors co-linear?}

Employing the analytical solution for $\eta, \lambda, R$ and $\phi$ given in (24), the closed-form expressions for the non-zero elements of the collisional stress tensor, $\boldsymbol{P}^{c}=\boldsymbol{\Theta}(m \boldsymbol{C})$, can be obtained whose eigenvalues have been calculated (not written here). The corresponding eigenvectors are

$$
\left|P_{1}^{c}\right\rangle=\left[\begin{array}{l}
1 \\
1 \\
0
\end{array}\right], \quad\left|P_{2}^{c}\right\rangle=\left[\begin{array}{c}
-1 \\
1 \\
0
\end{array}\right], \quad\left|P_{3}^{c}\right\rangle=\left[\begin{array}{l}
0 \\
0 \\
1
\end{array}\right],
$$

which coincide with the eigen-directions of the shear tensor, $\boldsymbol{D}=\left(\boldsymbol{\nabla} \boldsymbol{u}+(\boldsymbol{\nabla} \boldsymbol{u})^{T}\right) / 2$, given in (13). Since (29) holds exactly at $v=v_{\max }$, we conclude that the eigen-directions of $\boldsymbol{P}^{c}$ and $\boldsymbol{D}$ are co-linear at $v=v_{\max }$, but they would be non-colinear when the density effects are taken into account for $v<v_{\max }$. The latter has been checked via the numerical solution of second moment equation [7, 8].

\subsubsection{Scaling relations}

We see from (25) and (26) that both pressure and viscosity scale with $g_{0}$ in the dense limit. Using Torquato's form [11] for 'equilibrium' hard-sphere system, we have

$$
\mu, p \sim g_{0} \sim\left(v_{\max }-v\right)^{-1} .
$$

This exponent is much smaller than 2 , found empirically from experiments and simulations on non-Brownian suspensions. (Leaving aside subtle many-body correlations, accurate data on how $g_{0}$ diverges near jamming is therefore important for any meaningful conclusion.) Among two normal stress differences, $\widetilde{\mathcal{N}}_{1} \rightarrow 0$ as $v \rightarrow v_{\max }$ but the second normal stress difference diverges like

$$
\widetilde{\mathcal{N}}_{2}=\left(P_{y y}-P_{z z}\right) \sim p \mathcal{N}_{2} \sim\left(v_{\max }-v\right)^{-1},
$$

(similar to the scaling of pressure) since its scaled version $\mathcal{N}_{2}$ remains finite at $v=v_{\max }$ (see Fig. 1).

It is reasonable to argue that the onset of jamming is associated with a diverging length scale, $L_{J} \sim\left(v_{\max }-v\right)^{-\alpha}$, with $\alpha \sim 0.73$ [12]. This can be tied with a lesser amount of collisional dissipation $\left(\mathcal{D} \sim L_{J}^{-1}\right)$ as $v \rightarrow v_{\max }$ and hence the temperature is likely to diverge as $T \sim\left(v_{\max }-v\right)^{-2 \alpha}$ which follows from the energy balance equation. Incorporating these informations into the expression of shear viscosity $\left(\mu \sim g_{0} L_{J} \sqrt{T}\right)$ yields an exponent for the viscositydivergence $\left[\mu \sim\left(v_{\max }-v\right)^{-\delta}\right]$ as $\delta=(1+2 \alpha) \approx 2.5$ which is very close to simulation results [13] of $\delta=2.58 \pm 0.1$ for frictionless systems. On the other hand, for $\alpha=1 / 2$, $L_{J} \sim\left(v_{\max }-v\right)^{-1 / 2}$ and $T \sim\left(v_{\max }-v\right)^{-1}$ which recovers the empirical scaling relation, $\mu \sim\left(v_{\max }-v\right)^{-2}$, for shearviscosity near jamming. These exponents can be verified in future simulations.

\section{References}

[1] N. Sela, I. Goldhirsch, J. Fluid Mech. 361, 41 (1998)

[2] J. T. Jenkins, M. W. Richman, J. Fluid Mech. 192, 313-328 (1988)

[3] M. Alam, S. Luding, J. Fluid Mech. 476, 69 (2003)

[4] M. Alam, S. Luding, Phys. Fluids 15, 2298 (2003)

[5] M. Alam, S. Luding, In Powders and Grains (Eds: R. Garcia-Rojo, H.J. Herrmann \& S. McNamara; A. A. Balkema, Netherlands, 2005), pp. 1141-1144.

[6] H. Grad, Commun. Pure Appl. Math. 2, 331-407 (1949)

[7] S. Saha, M. Alam, J. Fluid Mech. 757, 251-296 (2014)

[8] S. Saha, M. Alam, J. Fluid Mech. 795, 549-580 (2016)

[9] S. Saha, M. Alam, Preprint (2017, to be submitted)

[10] S. Savage, D. Jeffrey, J. Fluid Mech. 110, 255 (1981)

[11] S. Torquato, Phys. Rev. E 51, 3170-3184 (1995)

[12] T. Hatano, Phys. Rev. E 79, 050301 (2009)

[13] P. Olsson, S. Teitel, Phys. Rev. Lett 109, 108001 (2012) 